\title{
CONF-8806117-3
}

CONF $-8806117=-3$

DE88 011842

\section{EXCITATION AND PHOTON DECAY OF GIANT MULTIPOLE RESONANCES - THE ROLE AND FUTURE OF MEDIUM-ENERGY HEAVY IONS}

F. E. Bertrand, J, R. Beene, and D. J. Horen

Oak Ridge National Laboratory, ${ }^{*}$ Oak Ridge, IN 37831-6368, U.S.A.

\section{DISCLAIMER}

This report was prepared as an account of work sponsored by an agency of the United States Government. Neither the United States Government nor any agency thereof, nor any of their employees, makes any warranty, express or implied, or assumes any legal liability or responsibility for the accuracy, completeness, er usefulness of any information, apparatus, product, or process disclosed, or represents that its use would not infringe privateiy owned rights. Reference herein to any specific commercial product, process, or service by trade name, trademark, manufacturer, or otherwise does not necessarily constitute or imply its endorsement, recommendation, or favoring by the United States Government or any agency thereor. The views and opinions of authors expressed herein do not necessarily state or reflect those of the United States Government or any agency thereof. 
EXCITATION AND PHOTON DECAY OF GIANT MULTIPOLE RESONANCES THE ROLE AND FUTURE OF MEDIUM-ENERGY HEAYY IONS

F. E. BERTRAND, J. R, EEENE, and U. J. HOREN

Oak Rlage Na:ienal Laboratory. "Oak Ridge, IN 378zj-6368, U.S.A.

Inelas:ie scattering of medium energy heayy ions provides very large cross sections and peak-to-continuum ratlos for excitation of giant resonances. For energles abrye about $50 \mathrm{MeV} /$ nucleon, giant resonances are excited primarily through Coulomb excitation, which is indifferent to isospin, thus providing a good probe for the study of isovector giant resonances. The extremely large cross sections avallable from heavy ion excitation permit the study of rare decay modes of the giant resonances. in particular. recent measurements have been made of the photon decay of giant resonances following excitation by 22 and 84 MeV/nucleon 10 projectiles. The singles results at $84 \mathrm{MeV} /$ nucleon yield peak cross sections for the iscscalar giant quadrunole resonance and the isovector giant dipole resonance of epproximately 0.8 and $3 \mathrm{barns} / \mathrm{sr}$. respectively. Data on the ground state decay of the isoscalar giant quadrupole and iscrector giant dipole resonances are presented and compared with calculations. Decays to low-lying excited states are also discussed. Preliminary results from an experiment to isolate the ${ }^{202} \mathrm{~Pb}$ isovector quadrupole resonance using its gama decay are presented.

1. INTRODUCTION

In 1972 the field of study of giant resonances which had been limited to the isovector Giant Dipole Resonance (IVGDR) was greatly broadened by the discovery in several nuclei of an isoscalar giant quadrupole resonance $(15 G O R) .^{1}$ These early observations were made through the use of inelastic scattering of electrons and protons rather than by the, to that time at least, conventional method of study of giant resonances, the photonuclear reactions. These observations led to similar studies in essentially every laboratory in the world that could produce a medium-energy light-ion beam and in a few years several new isoscalar giant resonances had been found in nuclei spanning the mass range of the periodic table. The gross properties of the various resonances (excitation energy, width, strength) have, in general, been wel! determined. As an example of this we show in table 1 a list of the giant rescrances observed in $20 \mathrm{SP}_{\mathrm{P}}$.

While the evidence for all these resonances is not uniformly strong, there is at least reasonable evidence for them. As far as the IVGOR, I5GOR, and

\footnotetext{
"Operazed by Martin Mariezta Energy Systems, Inc. under contract
}

DE-ACO5-S4OR21400 with the U.S. Department of Energy.

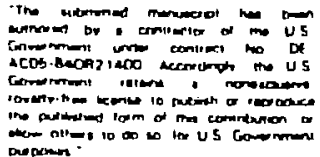


Table !

Gian: Resonances in $208 \mathrm{pb}$

\begin{tabular}{|c|c|c|c|}
\hline resconance & $15 / 11$ & $\begin{array}{c}\text { Excitation } \\
\text { Energy }\end{array}$ & $\begin{array}{l}\text { Designation } \\
\text { in this Paper }\end{array}$ \\
\hline $\begin{array}{l}\text { Dipole } \\
\text { Dipole } \\
\text { Quadrupole } \\
\text { (Ouadrupole) } \\
\text { Monopole } \\
\text { Monopole } \\
\text { Octupole } \\
\text { Hexadecapole }\end{array}$ & $\begin{array}{l}\text { IV } \\
\text { is } \\
\text { is } \\
\text { iv } \\
\text { is } \\
\text { iv } \\
\text { is } \\
\text { is }\end{array}$ & $\begin{array}{l}13.5 \mathrm{MeV} \\
17 ? \\
10.6 \\
22 ? \\
13.9 \\
32 \\
20 \\
12\end{array}$ & $\begin{array}{l}\text { IVGDR } \\
\text { ISGDR } \\
\text { ISGQR } \\
\text { ISGOR } \\
\text { I SGMR } \\
\text { IVGMR } \\
\text { ISGOR } \\
\text { ISGHR }\end{array}$ \\
\hline
\end{tabular}

ISGMR resonances are concerned, data from virtually all hadron measurements are in agreement with one another and are in agreement with the newest (e, $e^{\prime} n$ ) measuremenes. Most of the resonances listed in Table 1 have been seen in a large number of nuclei and the systematic variation of the energy of a given resonance with nuclear mass helps to provide evidence for the proper identification of a peak as a giant resonance.

While much has been learned about new giant resonzices during the past 15 years there are still many open questions. For example, while there is good evidence for many isoscalar resonances, there is, except for the IVGOR, meager evidence for the existence of isovector, electric giant resonances. An exception to this statement is the IVGMR which has been found in a number of nuclei using the pion charge exchange reaction. 2 There is very little data on high multipclarity (L>3) resonances of isoscalar or isovector character. These rescnarices are likely to be very broad and, therefore, be difficult to cbserie excerimentally. Finally, there is very little experimental information on the microsccpic nuclear structure of the giant resonances or on the interaction of these scecial high-excitation energy collective states with the very high density cf levels in the nuclear continuum from which these special states arise (this is to say. information on the demping of the giant resonance into the nuclear continuum).

In this paper we discuss the ways that the use of medium-energy heavy ions to excite giant multipole resonances will help address some of the questions raised above. It is the onset of Coulomb excitation dominance of the excitation mechanism that provides extremely large cross sections for excitation of giant resonances of BorH isoscalar and isovecior characker. These cross sections are realized without a compensatingly large increase in the underlying 
nuclear continuum, thus leading to extraordinarily large peak to continuun. ratios. The large cross sections and large peak-to-continuum ratios make $t$ possitle to carry out detalled gamma-ray spectroscopy of the giant multipole resonances thereby opening up the possibility of understanding some of the microscooic properties of the resonanres and their interaction with the continuum. We believe that it is in the study of very low cross section decay processes anc in the study of isovector states that the use of medium-energy reayy ions will have their greates: impact on the giant resonanze field. Ir in is oresenta:ion we emphasize those aspects of the stucy of giant resonances wich are unique to the use of Nucleus-Nucleus interaction, the topic of this conference.

While we will discuss in our limited tine the excitation and photon decay of cipole and quadrupole (isnvector and isoscalar) states we point out that other types of stucies of giant resonances will benefit from the use of the large cross sections from medium eneray heavy ion scattering. For example, the search ${ }^{3}$ for multi-ohonon resonance states has already provided some provocative resul:s from inelastic scattering of heavy-ions. The understanding of the discrepancy otween seemingly similar measurements ${ }^{4} .5$ searching for very high excitation states should be a challenge to those of us in the field. Other types of decay measurements will also berefit greatly from the large cross sections and peak to continuum ratios. The neutron decay studies such as those carried out at Groningen and ORNL (see contributions to this conference) will zurely benefit.

\section{HEAYY-ION EXCITATION}

How then does inelastic scattering of heavy ions fit into this picture? There have been several ways suggested that heavy ions might enhance the study of giant resonances: by providing larger cross sections, a larger peak-tocontinuum ratio due to a decrease of knock-out reactions, and the possibility to selectively excite resonances of high angular momentum. An example ${ }^{6}$ of some of the first studies of heavy ion excitation of giant resonances is shown on figure 1 and indicates that none of the hoped for gains were realized. These spectra are from $200 \mathrm{MeV}(-17 \mathrm{MeV} /$ nucleon) $12 \mathrm{C}$ inelastic scattering from $208 \mathrm{pb}$ and are shown along with a spectrum from $120 \mathrm{MeV}$ alpha particle ineiastic scattering from the same target. In all the data a peak is observed at about 10.6 MeV, the energy of the ISGQR. The $-3 \mathrm{mb} / .5 \mathrm{r}$ cross section for the ISGOR in the ${ }^{12 C}$ spectrum is consiaerably less (- tactor of ten) than that obtained for $120 \mathrm{MeV}$ aloha particle scattering. Furthermore, the peak-to-continuum ratio for the 1SGQR in the carbon reaction is considerably poorer than that for the 


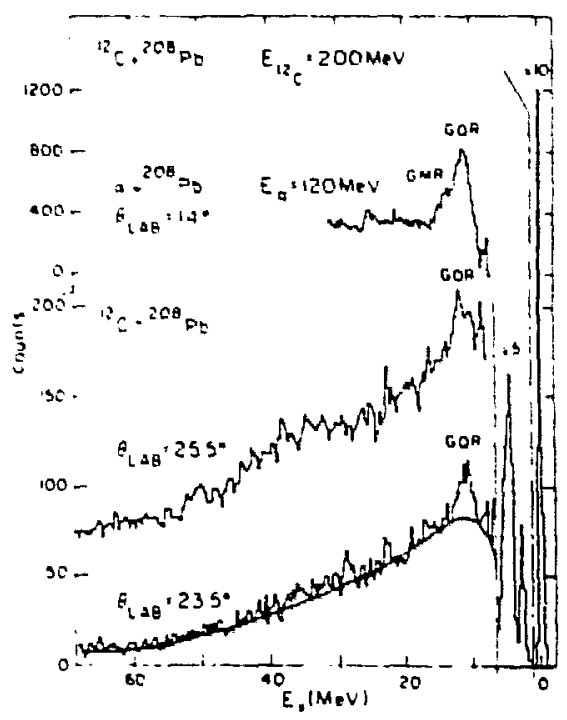

F!GURE I

Spectra of the "Po (12C, ${ }^{12} \mathrm{C}$ ) $)^{205 \mathrm{~Pb}}$

reactions at $E_{12} \mathrm{C}=200 \mathrm{MeV}$ and $208 \mathrm{po}\left(a, a^{\prime}\right) 203 \mathrm{pb}$ at $E_{\mathrm{C}}=120 \mathrm{MeV}$ (Ref. 6).

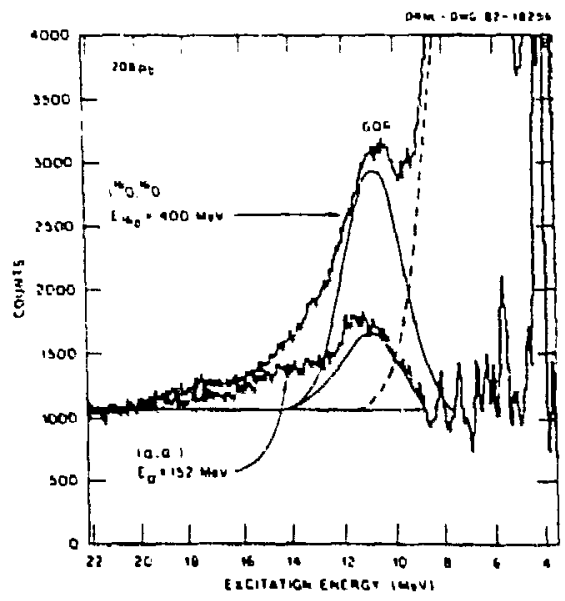

FIGURE 2

Comparison of $209 \mathrm{~Pb}$ giant resonance spectra as obtained from the $\left(160.160^{\circ}\right)$ reaction at $400 \mathrm{MeV}$ and the $\left(a, a^{\prime}\right)$ reaction at $152 \mathrm{MeV}$. The spectra are normalized at 22 MeV (Ref. 7).

aloha particle spectrum. Ciearly there is no enhancement of other, high multipolarity resonances in these heavy ion spectra. Overall these results could be considered diseppointing.

More recently, accelerators have been able to provide heavy ions in the energy range of 20-40 MeV/nucleon and experiments have been performed to excite giant resonances with those higher energy beams. It had been anticipated that the cross section for heavy-ion excitation of giant resonances should increase rapidly with increasing beam enargy. In figure 2 we show spectra from the $\left(160,160^{\prime}\right)^{7}$ and $\left(a, a^{\prime}\right)^{8}$ reactions on ${ }^{208 \mathrm{~Pb}}$ at $400 \mathrm{MeV}$ and $152 \mathrm{MeV}$ respective:y. At 25 MeV/nucleon the giant resonance spectrum is much more prominent than it is at the lower heavy-ion energies. The cross section at the grazing angle is $-40 \mathrm{mb} / \mathrm{sr}$. Comparable to the $\left(a, a^{\prime}\right)$ cross section but the peak-tocontinuum ratio is much larger for the heavy-ion reaction than for the alpha particle reaction. However, note in the oxygen spectrum the large rise in cross section at excitation energies just below the of the quadrupole resonance. This large peak arises from exitation of states in the 160 projectile and foints out one of the serious problems with heavy-ion inelastic excitation 
of giant resonances; exfitation of the projectlle and nucleon plckup and subsequen: nuclean desay of the projectile. It is possible to eliminate or at least substantially raduce contamination of the heavy ion inelastic spectra from projectile excttation by choosing a projectile that is unbound to neutron emission a: very low energy, projectiles such as ${ }^{13} \mathrm{C}$ or 170 . Figure 3 show four inelastic scattering spectra ${ }^{4}$ from 208po. Figure 3b shows a $\left(P . D^{\prime}\right)$ spectrum ${ }^{9}$ using $334 \mathrm{MeV}$ protons taken with $\rightarrow 70 \mathrm{keV}$ energy resolution while figure $3 \mathrm{c}$ shows the 160 spectrum of figure 2 . The spectrum in figure $3 \mathrm{a}$ is from inelastic seattering of $376 \mathrm{MeV} 170$ and does not show the large peak at abou: 6-8 MeV from projectile excitation as is the case for 160 since 170 is neutron unstable at about $4 \mathrm{MeV}$. The proton and 170 spectra are almost identical except for the region of the IVGDR. Finally, figure 3d shows the inelastic spectrum from $22 \mathrm{MeV} /$ nucleon 325. Now one sees considerable structure in the giant resonance region that apparently arises from projectile not target
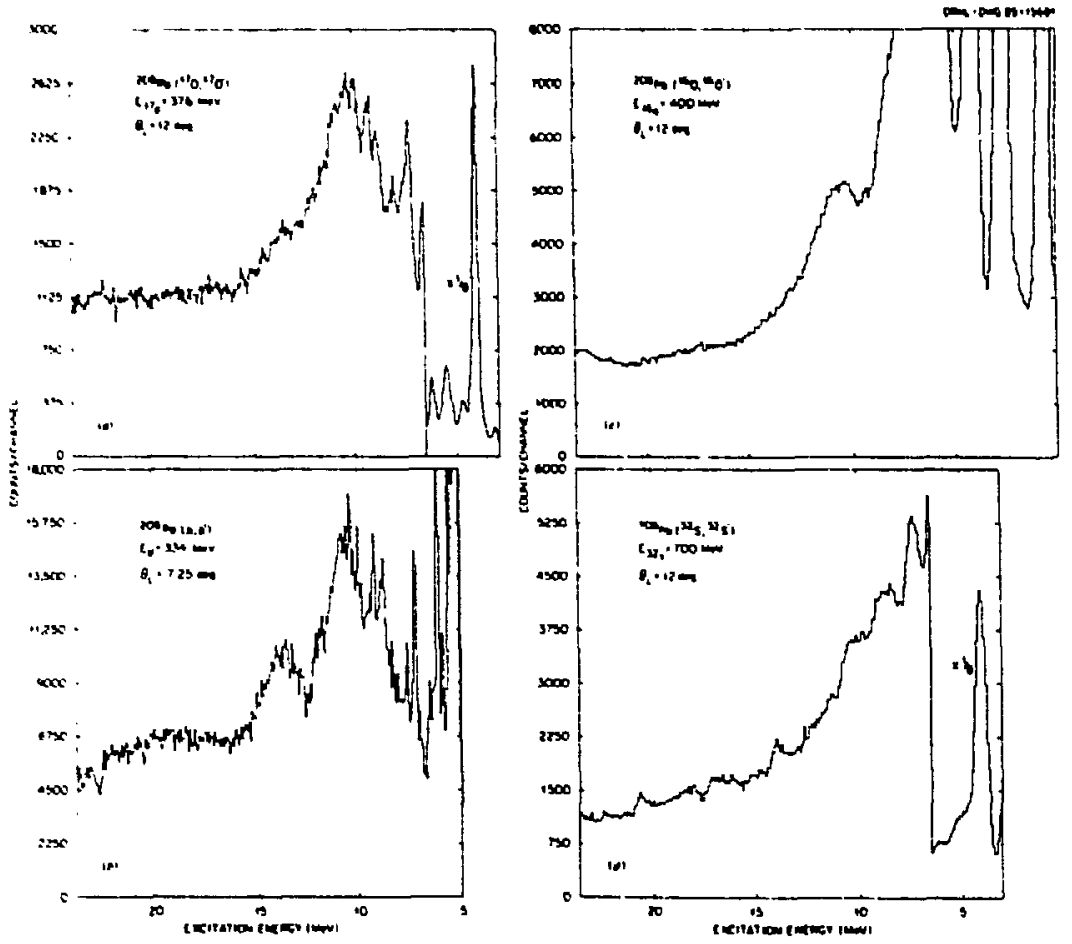

FIGURE 3

Inelastic scattering spectra for excitation energies between -3 and $-24 \mathrm{MeV}$. (a) $\left(170.170^{\circ}\right), 12^{\circ},(0)\left(p, p^{\prime}\right), 7.25$ (Ref. 9), (c) $\left(160,160^{\prime}\right) 12^{\circ}$ (Ref. 7), and (d) $\left({ }^{32} S^{32} 5^{\prime}\right), g^{\circ}$ (Ref. 4). 
effects. These spectra demonstrate that care must be exercised in the choice of the heavy ion projectile used, and the energy of the heavy ion should also de considered to ensure that the excitation energy region of interest is not compromised by projectile effects. These problems with heavy ion scattering are often difficult to detect and can lead to incorrect conclusions concerning the excitation of new states in nuclei.

While use of heavy ion inelastic scattering in the energy range of 20-30 MeV/nucleon clearly offers large cross sections and enhanced peak-to-continuum ratios, and proper choice of projectlle can eliminate many of the effects of projectile excitation, it is still a fair assessment that heavy-ion inelastic scattering measurements for projectile energies up to -40 MeV/nucleon show a selectivity similar to that observed with lighter probes. This is to say that the spectra are dominated by excitation of the ISGQR and the ISGMR. Furthermore. identification of the multipolarity of the giant resonance is difficult because the angular distributions for different angular momentum transfers in inelastic heavy-ion scattering are so similar.

Can we then expect any future for heavy.ion excitation of giant resonances? The answer lies in the use of still higher energy heavy ions where the effects of Coulomb excitation become dominant over the nuclear interaction. Coulomb excitation by high-energy heavy ions will provide, as we shall see below, very large differential cross sections for inelastic excitation of the resonances. Furthermore, Coulomb excitation operates equally well cn isoscalar and isovector states providing the possibility that for the first time, hadron scattering can provide strong excitation of isovector giant resonances.

If we consider the Coulomb excitation process in terms of the rapidiy changing Coulomb field created at the target by a projectile passing at high velocity in terms of the equivalent virtual photon field, then the couiomb excitation process is equivalert to the absorption of the virtual photons by the nucleus. In figure 4 we show intensity spectra for electric dipole virtual photons as seen by a $208 \mathrm{pb}$ target for a range of 160 incident energies between 50 and $1500 \mathrm{MeV} /$ nucleon. At the lowest incident energies the virtual photon spectrum drops rapidly with increasing excitation energy and clearly shows why Coulomb excitation with low-energy projectiles is limited to the study of lowlying states. On the other hand, the spectrum at $1500 \mathrm{MeV} /$ nucleon is considerably flatter in the excitation energy range of 10-50 MeV/nucleon.

To make these calculations more relevant to experimental observation we show in figure 5 differential cross sections for excitation of the 15GQR in 208pb for several incident energies of 170 . At the lower energies, 25 and 50 MeV/nucleon, the cross section is mainly from the nuclear interaction, while at the higher energies coulomb excitation dominates. The effect of the increasing 


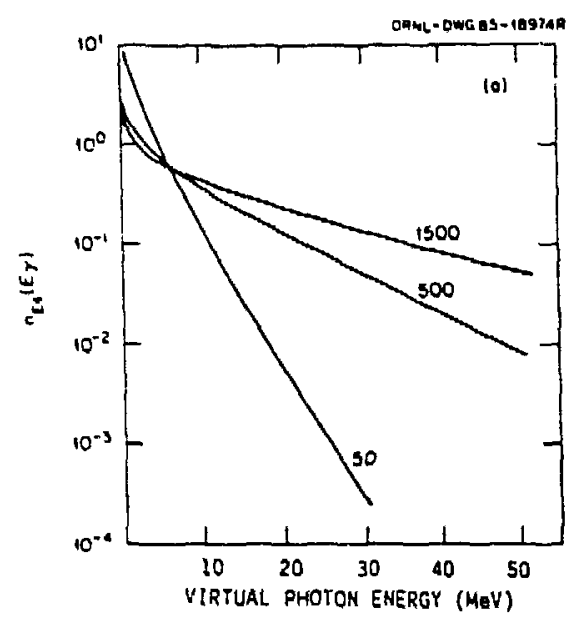

FIGURE 4

El virtual photon intensity spectrum seen by a $208 \mathrm{~Pb}$ target due to 50,500, and $1500 \mathrm{MeV} /$ nucleon 160 beams.

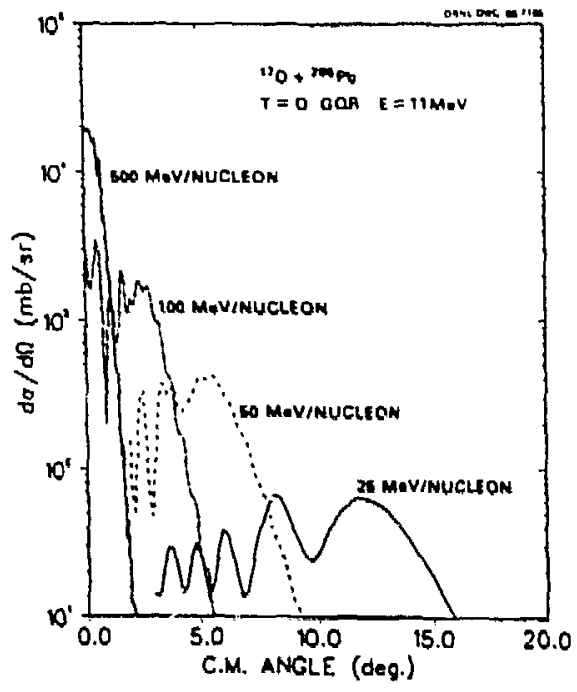

FIGURE 5

Calculated angular distributions for the isoscalar glant quadrupole resonance excited by inelastic scittering of various energy 170 ions.

importance of coulomb excitation with increasing incident energy 15 dramatic. The peak cross section for excitation of the ISGQR increases by over two orders of magnitude between $25 \mathrm{MeV} / \mathrm{nucleon}$ and $500 \mathrm{MeV} / \mathrm{nuclean}$, and would continue to increase at even higher energies.

Perhaps even more dramatic is the effect of the Coulomb excitation on an IVGQR at $22 \mathrm{MeV}$ in 208po as shown in the calculated differential cross sections on figure 6 . The nuclear excitation of the isovector states proceeds through the isospin part of the nucleon-nucleus interaction which is quite weak in compariscn with the strength of the non-spin and non-isospin parts. On the other hand, the Coulomb exctitation is as strong for isovector states as it is for isoscalar states, all other things being equal. At $25 \mathrm{MeV} / \mathrm{nucl}$ eon the maximum eross section expected for the IVGQR is $\sim 0.5 \mathrm{mb} / \mathrm{sr}$, compared to a measured cross section of about $60 \mathrm{mb} / \mathrm{sr}$ for the ISGQFi. On the other hand, at 500 MeV/nucleon where the Coulomb excitation is dominant, the cross section for the IVGQR is calcuiated to be $10,000 \mathrm{mb} / \mathrm{sr}$ !

The beam energies at GANIL should be high enough to provide a glimpse of the large Coulomb excitation cross sections. We have been involved in a research pragram at GANIL with collaborators from GANIL, Saclay, Strasbourg, and Illinois in measurements of the excitation and photon decay of giant resonances excited by $84 \mathrm{MeV} /$ nucleon 170 beams. Measurements ${ }^{10}$ were made using the 84 
MeV/nucleon 170 beam (full energy for 170 ) from the GANIL facility. Inelastically scattered 170 ions were detected and identified in the energy loss magnetic spectrometer \$PEG. The overall energy resolution was about Eoc keV due in large part to the use of a $5.1 \mathrm{mg} / \mathrm{cm}^{2} 203 \mathrm{po}$ target. The spectrograph was set to accept events in the angular range from 1.5 oegrees to 5.0 degrees. Figure 7 shows a spectrum from the GANIL measurements (solid line) comparad to a spectrum, al ready shown in figure 3a, from $22 \mathrm{MeV} /$ nucleon measurements at ORNL. The lower energy date is normalized to the GANIL data in the unstructured nuclear continuum (about $40 \mathrm{MeV}$ ). There are several obviously outstanding features in the GANIL data. First the giant resonance peak is nuge, rising almost a factor of 10 above the continuum. Reference to figure $3 a$ shows that the $22 \mathrm{MeV} /$ nucleon spectrum rises over a factor of two above the continuum as does the proton spectrum shown in figure $3 b$. Clearly, one of the oojectives of the use of heavy lons for giant resonance studies, the possibility of enhancing the peak-to-continuum ratio, has been realized. It is also apparent from figure 7 that the excitation energy of the centroid of the giant resonence peak in the GANIL data is higher thain in the lower energy data. As will be shown below this is due to the fact that the Couloinb excitation of the

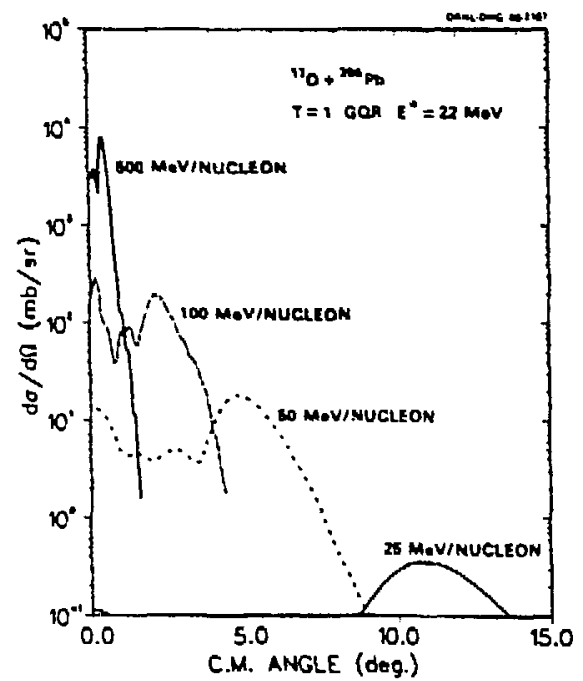

FIGURE 6

Calculated angular distributions for excitation of the isovector gient quadrupole resonance in $208 \mathrm{pb}$ by inelastic scattering of 170 ions of several different energies.

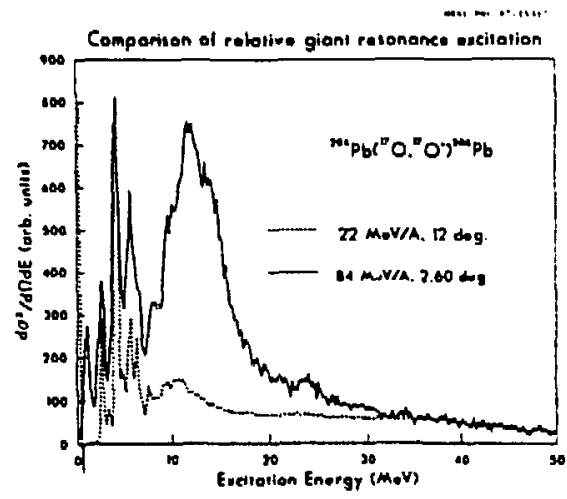

FIGURE 7

Spectra from inelastic scattering of 84 (Ref. 10) and 22 (Ref. 4) Mevi nuelean 170 from 208pb. The two spectra are normalized in the unstruetured continuum near $40 \mathrm{MeV}$. 
IVGOR dominates the ISGQR at the higher bombarding energy while the quadrupole resonance is the strongest excitation in the lower bombarding energy data. Note that for the GANIL data shown in figure 7, the giant resonance peak and the peaks from excitation of low-lying states are on the same arbitrary cross section scale. The peak height of the giant resonance structure is as large as that for the $4.08 \mathrm{MeV}, 2^{+}$level and larger than that foi the $2.6 \mathrm{MeV}, 3^{-}$level.

At incident heavy ion energies of 50-100 MeV/nucleon the Coulomb excitation probability drops very rapidly with increasing excitation energy, changing by over a factor of two across a peak as broad as the IVGOR. Thus, the shape of the IVGOR peak is considerably altered -he excitation mechanism from that observed in photonuclear work. Figure $u$ shows the effect of the reaction mechanism on the shape of the IVGOR for excitation by $84 \mathrm{MeV} /$ nucleon (dotted line) and $22 \mathrm{MeV} /$ nucleon (dash-dot line) 170 incident ions. The solid curve shows the shape of the IVGDR as taken from photonuclear measurements. 11 As expected, the effect is more dramatic at the lowir energy. We have used the shape as shown in figure 8 in the analysis of the data from GANIL and from ORNL. The shape of the IVGDR as altered by the coulonb interaction changes with angle.

Figure 9 shows spectra from the GANIL measurements at three angles decomposed into peaks from the ISGQR, IVGDR, and ISGMR with centroids and widths taken from previous measurements and for the IVGOR, from the shape shown on figure 8. The areas of the peaks were allowed to vary and the shape of the underlying continuum was taken as generally flat under the peak. It is comforting to be able to say, for perhaps the first time, that, at least at most angles. the assumptions made about the shape and magnitude of the continuum underlying the giant resonances do not dominate the uncertainty in the data. The cross sections for the ISGMR and the IS giant hexadecapole resonance are quite sinall relative to the cross section for the dipole and quadrupole resonances and for that reason are quite uncertain and will not be dwelled upon in this discussion except to note that the cross sections are consisient with previous measurements.

At most angles, excitation of the IVGOR dominates the spectrum. The cross section for the IVGDR reaches a magnitude of nearly 3 barns/sr which is about four times the peak cross section of about $800 \mathrm{mb} / \mathrm{sr}$ for the ISGOR. These huge cross sections show that we have realized another hoped for attribute of heavyion excitation of giant resonances - very large cross sections. The calculation shown as the solid line on the dipole data was generated assuming only Coulomb excitation of the IVGOR and the magnitude corresponos to $110 \%$ of the EWSR between 7 and $18.9 \mathrm{MeV}$. In the same energy range, the photonuclear reaction 


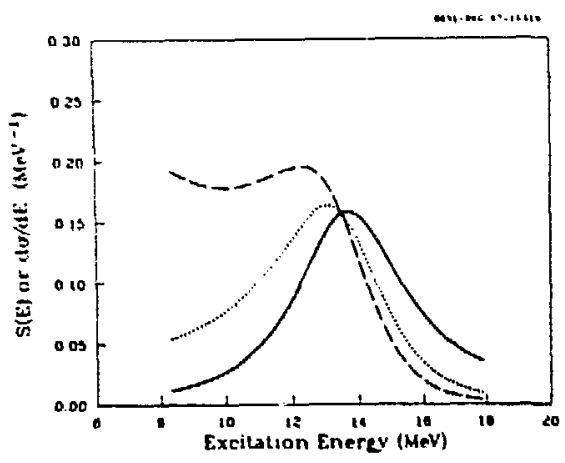

FIGURE 8

This figure shows the effect the rapidly changing coulomb excitation probability as a function of excitation energy has upon the shape of the 200pb IVGOR. The solid curve is the dipole shape from photonuclear reactions. The dotted curve is the shape expected from excitation by $84 \mathrm{MeV} / \mathrm{nucleon} 170$ ions and the dash-dotted curve is the shape expected for excitation by $22 \mathrm{MeV} / \mathrm{nucleon} 170$ ions.

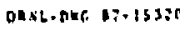

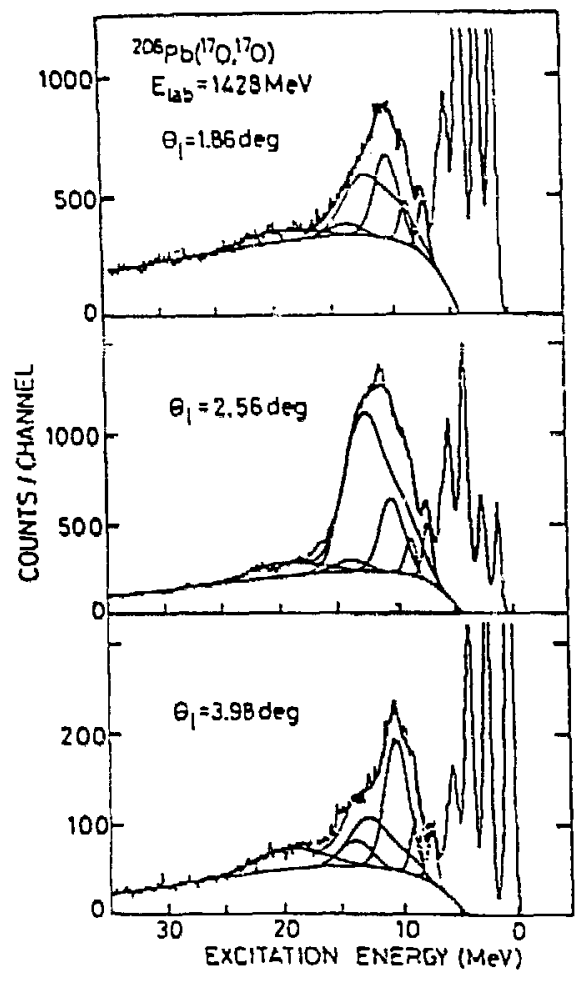

FIGURE 9

Ineiastic scattering spectra at $\theta_{\text {lab }}=1.86^{\circ}, 2.56^{\circ}$, and $3.98^{\circ}$ from the 208po(170,170') reaction at $1428 \mathrm{MeV}$. The solid curves show a decomposition of the spectra into resonance peaks at $-10.6 \mathrm{MeV}$ (ISGOR), $-13.6 \mathrm{MeV}$ (IVGDR), $14 \mathrm{MeV}$ (ISGMR), 7.5 MeV, 9.1 MeV and a broad, undefined, peak centered at $-20 \mathrm{MeV}$ and an underlying continum (Ref. 10).

yields about $103 \%$ of the EHSR. Wis thus, account very well for the dipole excitation with the Coulomb interaction and resonance parameters from photonuclear data. 11 The calculation for the 15GOR assumes both coulomb and nuclecr excitation as shown by the separate curves plotted on figure 10. The angular distribution is we!! described by the $L=2$ calculation with a deformation length of $0.49 \mathrm{fm}$ corresponding to exhaustion of $60 \%$ of the EWSR, in good agreement with previous data. The combination of excitation of the ISGQR via both coulamb and nuclear interactions provides for a broader angular distribution than is observed for the dipole where only coulomb excitation is indicated. 


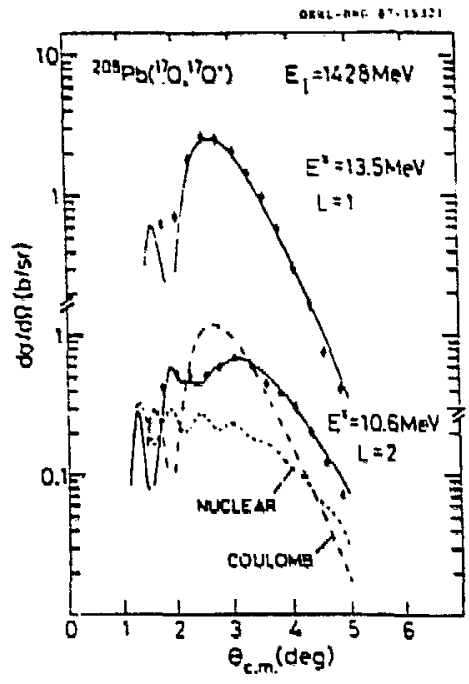

FIGURE 10

Experimental and calculated angular distributions for the isovector glant dipole resonance (upper part) and the isoscalar giant quadrupole resonance (lower part) in $208 \mathrm{pb}$. For the ISGQR, the calculated Coulomb and nuclear contributions to the cross section are shown (dashed lines) (Ref. 10).

\section{PHOTON DECAY}

Coincidence experiments designed to investigate decay properties now make up a significant part of the experimentul study of gluit resonances. Since most glant resonances (GR) lie above the particle emission threshold the dominant decay mode is usually nucleon or alpha emission (neutron emission in heavy nuclei) with gama decay having a small ( $\left.\leq 10^{-3}\right)$ probability. Nucleon emission can it: principle carry important information about the microscopic structure of resonances, damping mechanisms and damping rates. Photon docay studies face difficulties resulting from the small probability of such events, yet they too can provide signiflcant information, often 1 lluminating aspects of the GR different from those probed by nucleon decays. As will be seen, the low probability of photon decay can be mitigated by the large GR cross sections and resonance to sontinuum ratios which can be achieved with inelastic scattering of selected heavy ions at energies above $-20 \mathrm{MeV} /$ nucleon and both the cross section and resonance to continum ratio rapidly increase with increasing bombarding energy.

Photon decay data can be extremely sensitive to GR multipolarity - thus compensating for a principal weakness in heavy ion scattering. For example, gamma decay back to the ground state following heavy ion scattering is certain to be dominated by $E l$ decay, therefore by the isovector giant dipole resonance (IYGDR). Under very favorable conditions ground state decay of the giant quadrupole resonance (GQR) can also be observed - but higher multipolarities in the 10 to $25 \mathrm{MeV}$ excitation energy region are extremely unlikely to contribute to the 9.5. decay. This is illustrated in rigure 11, which shows the ground 
state gamma width $\left(\Gamma_{10}\right)$ expected for a sharp state exhausting $100 \%$ of the relevant isoscalar or isovector energy weighted sum rule (EWSR) as a function of multipolarity and energy, relative to that for the IVGDR. Ground state gamma decay can also yield data on the electromagnetic strength of resonances, and provide simple, well-defined conditions under which we can investigate the multistep theory of nuclear reactions in terms of which GR decay is conventionally discussed.

Photon decays from the GR to low-lying excited states are also potential sourees of significant information. These data, like ground-state decays, provide significant multipole selectivity - but are not limited to $L=1$ and 2 resonances. Decays to excited states, like ground state decay, are dominated by El transiticns. Thus, for example, transitions from the GR region to a low lying $5^{-}$state in an even-even nucleus is clear evidence for high spin $\left(4^{+}\right.$or $\left.6^{*}\right)$ strength. Decays to low-lying collective states can provide important information about the coupling of (isovector) GR modes to these low frequency collective modes. Recent calculations ${ }^{12,13}$ have shown that El transitions between isoscaiar GR and low lying collective states (also isoscalar) can be strongly suppressed. Thus, study of such transitions can provide important ata on the isospin character of resonances.

We have carried out studies of the photon decay of gtant resonance states at ORNL using $22 \mathrm{MeV} /$ nueleon 170 beams and at GANIL using $84 \mathrm{MeV} /$ nucleon beams. The purpose of the lower energy measurements was to study the decay of the ISGQR while the nigher energy studies were undertaken to study the decay of isovector resonances primarily the IVGOR. Figure 12 shows the cross sections expected for the various resonances at the energies used. At $22 \mathrm{MeV} / \mathrm{nucl}$ eon the ISGOR should dominate the excitation of the IVGOR and the IVGOR will be unobservable. However, at $84 \mathrm{MeV} /$ nucleon where Coulomb excitation plays a major role the IVGDR now is larger than the ISGQR and the cross section for the IVGOR is as large as that for the ISGOR at $22 \mathrm{MeV} /$ nucleon. Reference to figure 11 shows that the width for El transitions is about 100 times larger than for E2 transitions. This fact coupled with the cross sections shown on figure 12 clearly shows that at 22 MeV/nucleon the photon from the ISGQR may be $-10 \%$ of those from the IVGDR while at 84 MeV/nucleon photons from the ISGOR will likely be unobservable.

The study of the photon decay from the giant resonances was carried out at GANIL by detecting the gama rays in $\mathrm{BaF}_{2}$ detectors clustered in groups of 7 or 19. in coincidence with the inelastically scattered 170 ions detected in the magnetic spectrograph, SPEC. For our most recent run 99 detectors were used. The detectors were right hexagonal crystals with face-to-face dimension of 


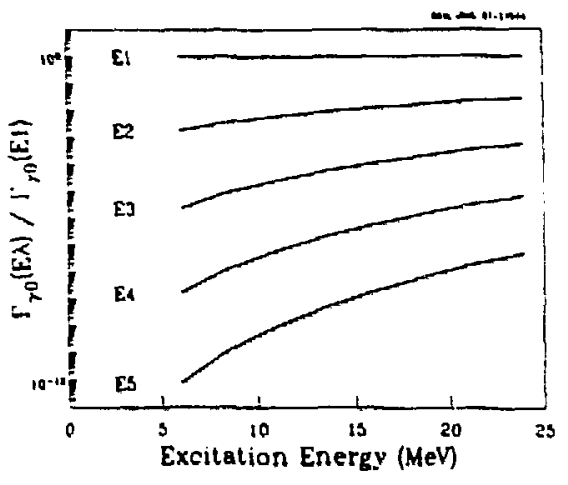

FIGURE 11

Ground-state gamma widths of hypothetical sharp states fully exhausting the appropriate isovector or isoscalar energy weighted sum rule as a function of the excitation energy of the state. relative to the El width.

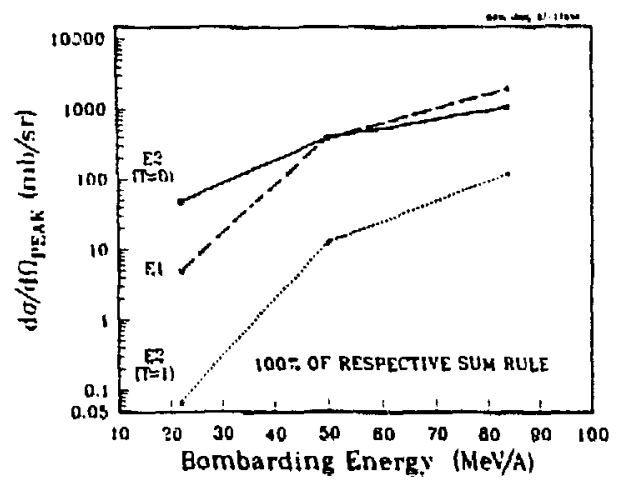

FIGURE 12

The peak differential cross section for inelastic scattering of 170 from $203 \mathrm{~Pb}$ to the resonance states ind $1-$ cated as a function of beam energy.

$8.7 \mathrm{~cm}$ and length $14 \mathrm{~cm}$ or $5.7-\mathrm{cm}$ by $20-\mathrm{cm}$ long. The elusters were placed at angles $(0,0)$ of $(70,172),(138,30),(138,50),(109,68),(109,187),(109,232)$, $(109,307),(109,352)$, and $(109,112)$. For the measurement ${ }^{14,15}$ of the photon decay at vak Ridge using 22 Mev/nucleon 170 ions, the gamas were detected in the 70 element Nal oali, the Spin Spectrometer, and the 170 ions in a ring of silicon surface barrier detectors. Figure 13 snows an inelastic scattering spectrum at 84 Mev/nucleon with the requirement of a coincidence with a single gamma ray to the ground state (i.e., with $E_{Y}=E^{*}$. where $E^{*}$ is the excitation energy). Figure 13 corresponds to a broad range of 170 angles from $2^{\circ}$ to $3.5^{\circ}$. The gamma coincidence spectrum shows prominent structures corresponding to well known low-lying states which have strong ground state gamma branches, notably the $2.6 \mathrm{MeV} \mathrm{3-}, 4.08 \mathrm{MeV} 2^{+}$states, and the $5.512 \mathrm{MeV} \mathrm{1-}$ states. Gama angular distributions demonstrate that most of the yield between the $5.5 \mathrm{MeV}$ state and the neutron separation energy $(7.4 \mathrm{MeV})$ corresponds to 1 - 5 tates (probatly the 7.06 and $7.08 \mathrm{MeV} 1^{-}$states), but a $2^{+}$state, at $-6.2 \mathrm{MeV}$ is also present. Above the neutron separation energ; the coillcident yield falls rapidly, but rises again to a strong broad peak in the GR region. The comments made earlier, together with the large cross sections for the IVGDR, would lead us to expect the $\Omega$ to $20 \mathrm{MeV}$ region of the gama coincidence spectrum to be dominated by the IVGOR. This is fllustrated qua:itatively in figure 14. The heavy solid line is the GR peak from the singles data. The short dashed curve is the distribution of IVGOR cross section calculated assuming pure Coulomb excitation, and utilizing the strength distritution for the IVGDR from photonuclear 


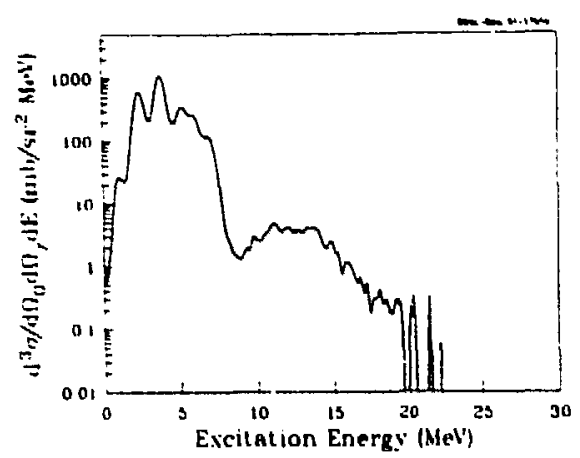

FIGIJRE 13

Inelastic spectrum in colncidence with gamma rays to the ground state. (The 170 angles are $\theta=2.0^{\circ}-3.5^{\circ}$ in this case, the gama detector angles are given in the text.)

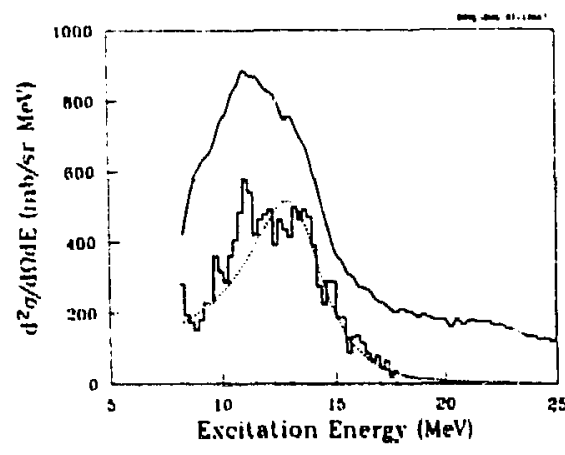

Figure 14

Multipole selectivity by gama coin. cidence. The heavy solid line is the giant resonance region of the $84 \mathrm{MeV} /$ nucleon data in figure 7. The dashed line is the theoretical contribution of the gient dipole resonance to the experimental spectrum. The histogram is the $1{ }^{1} 0-Y_{0}$ coincidence data of figure 13.

experiments. The histogram is the gamma coincidence spectrum, arbitrarily normalized. Clearly, the ground state garma coincidence effectively selects the IVGOR fro.n the complex multipole distribution present in the singles spectrum. This fact is illustrated more quantitatively in figures 15 and 16 which show $170-\gamma_{0}$ angular correlations. In figure 15 the correlation is displayed as a function of 170 angle, for ground state gammas at $\theta=90^{\circ}, \phi=270^{\circ}$, while in figure 16 the data are aisplayed as a function of $y$ angle for the 170 ions detected in the range $\theta\left({ }^{17}\right)=2^{\circ}$ to $3.5^{\circ}$. In this case the angle $\theta_{y}$ is referred to the direction of the 208pb recoil rather than the beam axis. The lines on figures 15 and 15 are ealculations carried out with the code ECIS, ${ }^{16}$ assuming pure Coulomb excitation of the IVGDF with its totai strength and distribution taken from Ref. 11. The calculation of the absolute theoretical cross sections in figure 15 involves an average ground state gama branching ratio, which is, of course, not provided by the ECIS calculation. For now this branching ratio can be regarded as determined by fitting the overall normalization in figure 15 to be $0.017 \pm 0.002$, however, we will see later that this quantity is in excellent agreement with a calculation containing no free parameters.

All IVGOR calculations shown assume pure coulomb excitation. Nuclear excitation of the IVGDR can play a role primarily through terms arising from the fact that both projectile and target have a neutron excess. Calculations employing a nuclear transition density calculated following the methods of 
Satchler, ${ }^{17}$ demonstrate that the nuclear contribution to the cross section of the IVGDR is about $10^{3}$ times smaller than the Coulomb, and has no significant effect on any of the calculations presented here.

The absolute yield of ground state galma rays can be calculated from the froperties of the IVGDR by applying the ideas of the multistep theory of nuclear reactions. 18,19 The collective $1 p-1$ h GR state is considered as a doorway state which couples strongly to the inelastic scattering procesa. This state damps into the more complex $2 p-2 h$. 3p-3h, etc. States eventually reaching the fully damped compound states. The cross section for emission of groundstate gamma rays following inelastic scattering can be expressed as:

$$
0_{x, x^{\prime} y_{0}}(E)=\sigma_{x, x^{\prime}}(E)\left(\sum_{i=1}^{\Gamma} \frac{\Gamma_{Y_{0}, i}}{\Gamma_{i}}\left[\prod_{j=1}^{i-1} \frac{\Gamma_{j}^{+}}{\Gamma_{j}}\right]\right) \text {. }
$$

${ }^{0} x_{,},(E)$ is the distribution of excitation cross section obtained from a DWBA calculation, the sum in parentheses runs over the hierarchy of levels of complextty from the doorway stage $(t=1)$ to the compound $\left(r^{\text {th }}\right)$ stage. The

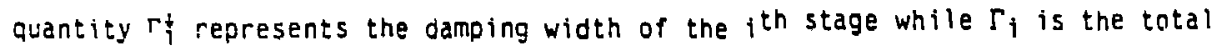
width. Application of this general expression requires a great deal of knowledge conceriling the various widths associated with each stage. For

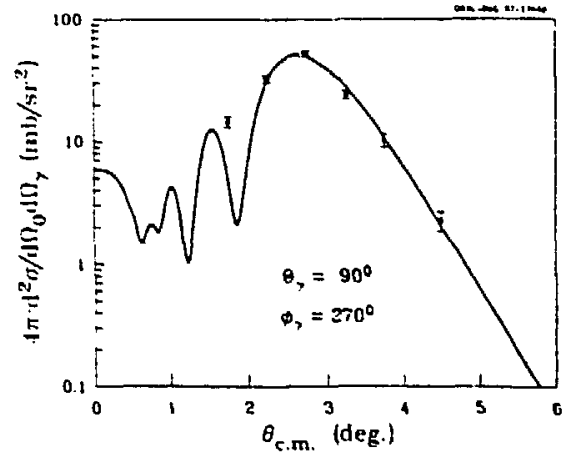

FIGURE 15

$170-Y_{0}$ angular correlation for the $208 \mathrm{po}(170,170$ ) reaction at $84 \mathrm{MeV})$ nucleon, for fixed $\gamma$ angle $\theta_{\gamma}=90^{\circ}$. $\phi_{Y}=270^{\circ}$.

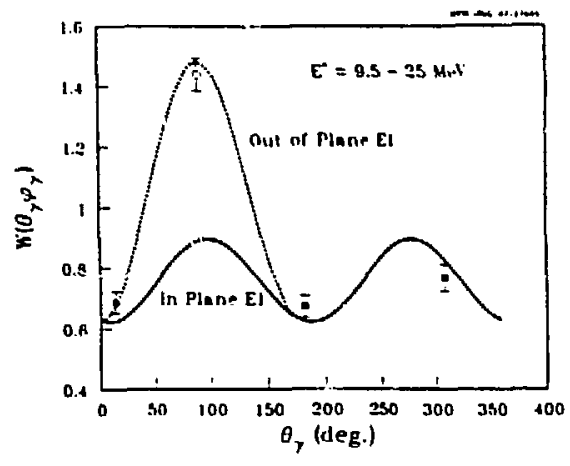

FIGURE 16

The same correlation as figure 15 , but for fixed 170 angle $\left(\theta=2^{\circ}-3^{\circ}\right)$ and varying $y$ angle. The lines are from theoretical calculations assuming pure Coulomb excitation of the GDR. Filled data points and the solid curve lie in the reaction plane $\left(\phi=0^{\circ}\right.$ and $\left.180^{\circ}\right)$. (For convenience the $\phi=180^{\circ}$ half plane is labeied by $\left.\theta+180^{\circ}.\right)$ The open point and dashed $l$ ine refer to the $\phi=270^{\circ}$ half plane. 
simplicity and without much justification, we will use a two stage approximation. considering only the GR doorway and the compound states, as:

$$
\sigma_{X, X^{\prime} y_{0}}(E)=\sigma_{x, X^{\prime}}(E)\left(\frac{\Gamma_{Y_{0}}}{\Gamma}+\left[\frac{\Gamma^{\downarrow}}{\Gamma}\right] B_{C N}(E)\right) .
$$

where the index 1 for the doorway state has been dropped. $\Gamma_{Y_{0}}$ can be calculated directly from the GR strength, $\Gamma$ is identified with the experimental width of the resonanze, and $B_{C N}(E)$ is the compound nucleus branching ratio. The quantity in square brackets assures that only that fraction of systems which survive the damping process ( $\Gamma^{+}$is the damping width) is included in the compound term. Theoretical and experimental results for $208 \mathrm{pb}$ indicate the $\Gamma^{+}$ $20.9 \Gamma$, so that this factor can be considered as introducing an uncertainty of up to $10 \%$ in the compound contribution (we will set it to unity). The compound branching ratio can be calculated from mean $Y_{0}$ and total widths obtained from Hauser-feshbach calculations, 20,21 but incluaing a correction for effacts due to the distribution of partiai widths which have been discussed extensively in the literature: 22 i.e..

$$
B_{C N}=C \frac{\left\langle\Gamma_{Y O}, C N\right.}{\left\langle\Gamma_{C N}{ }^{\prime}\right.}
$$

where $C$ is calculable in a straight forward way ${ }^{21}$ if we assume the partial widths have a Porter-Thomas distribution. The results of a calculation using Eq. (2), and the properties of the IVGDR $\left(\Gamma=4.0 \mathrm{MeV}\right.$ and $E_{0}=13.5 \mathrm{MeV}$ and strength $=100 \%$ of classical EWSR between 8 and $25 \mathrm{MeV}$ ) from Ref. 11 are compared with y-angle-averaged experimental data in figure 17 . The contribution of the first and second terms of EQ. (2) are shown separately as dashed and dash-datted curves respectively, and the sum as a solid line. The branchirg ratio, integrated over excitation energy from 9.5 to $25 \mathrm{MeV}$, is found to be 0.016 , in excellent agreement with the value $0.0 .17 \pm 0.02$ found earlier in connection with the data in figure 15. Furthermore the distribution of $\gamma_{0}$ yield as a function of excitation energy is reproduced very well.

Experimental details of the 22 Mev/nucleon measurements can be found elsewhere. ${ }^{14,15}$ at this energy the inelastic singles spectrum $\left[\theta(170)=13^{\circ}\right]$ is dominated by the isoscalar giant quadrupole resonance (ISGOR), with a cross section of about $40 \mathrm{mb} / \mathrm{sr}$ while the IVGCR is calculated to have a cross section $<3 \mathrm{mb} / \mathrm{sr}$. In figure 18 the total ground state gamma coincidence spectrum is shown. Comparison with figure 13 shows the much smaller yield of high energy gamma radiation in this case. Figure 19 shows the GR region of figure 18 , 
compared with a calculation identical to that discussed above in connection with figure 17. In figure 19a the total experimental spectrum is shown compared to a calculation including only the IVGDR, which, in spite of its weak excitation is clearly dominant. In figure 190 data obtained by subtracting the calculated IVGDR contribution from the experimental spectrum is compared with a calculation for the ISGQR garma decay. The ISGQR resonance parameters are taken from high resolution $\left(p, p^{\prime}\right)$ data. ${ }^{9}$ In both figure $19 a$ and $19 b$ the doorway and compound terms are shown separately as dash-dot and short-dashed curves respectively. whila the heavy solid lines are the sum. The light solid line in figure 19a represents the tail of the yield from around the neutron separation energy, while in figure 19b the light solid line represents the contribution of narrow $2^{+}$states found at 8 and $9.3 \mathrm{MeV}$ in $\left(p, p^{\prime}\right) .^{22}$ The gama ray angular correlation data shown in figure $19 \mathrm{c}$ confirms the presence of 22 gamma radiation in the 9 to $11 \mathrm{MeV}$ region and agrees very well with the relative $E 2$ and El contribution predicted by the calculations. From the data in figure $19 \mathrm{~b}$ we obtain a total E2 ground state cross section of $17 \pm 4 \mathrm{\mu b} / \mathrm{sr}$. corresponding to a total branching ratio for the ISGQR of $(4.1 \pm 1.0) \times 10^{-4}$ or an electromagnetic reduced matrix element of $B(E 24)=(6.2 \pm 1.2) \times 10^{3} e^{2} \mathrm{fm}^{4}$ for the $10.6 \mathrm{MeV}$ ISGQR. This corresponds to $87 \pm 20 \%$ of the strength expected for a state exhausting the full E2 EWSR, assuming the ratio of neutron to proton matrix elements in the ISGQR is $M n / M p=N / Z=1.5$. An experimental value for the ratio $M_{n} / M_{0}$ can be deduced from our $B(E 2 t)$ if the cross section for alpha-particle inelastic scattering 23 is used as a measure of $\left(M_{n}+M_{p}\right)^{2}$. We obtain $M_{n} / M_{p}=1.35 \pm 0.33$ in good agreement with the value $N / Z$ expected for ail approximately isoscalar GR having equal neutron and proton jeformation. Electron scattering results ${ }^{24}$ can be combined with $\left(a, a^{\prime}\right)$ results in a similar way to obtain $M_{n} / M_{\tilde{p}}-1.75 \pm 0.4$, again in good agreement with $N / Z$. These results disagree with the sinall value of $B(E 2 t)=(1.01 \pm 0.6) \times 10^{3} e^{2} \mathrm{fm}^{4}$ and $M_{n} / M_{p}=3.6$ deduced from recent $\pi^{+}$and $\pi^{-}$scattering data. 25 These quantities would require that the $10.6 \mathrm{MeV} \mathrm{GQR}$ in 208Pb have a strongly mixed isospin character.

It has been realized for some time that gamma decay of GR to low-lying collective states should carry important information about the coupling of low frequency surface vibrations to the GR. Such experiments on the IVGDR are an important part of the research program at tagged photon facilities. We have studied the branching to low-lying excited states in $208 \mathrm{pb}$ as a function of excitation energy between $\sim 8.5$ and $16 \mathrm{MeV}$ in our $22 \mathrm{MeV} /$ nucleon 170 scattering experiments at 0ak Ridge. Figure 20 shows the relative strength of gama-ray branches to a number of low-iying states. Figures 200 and 200 are for direct 

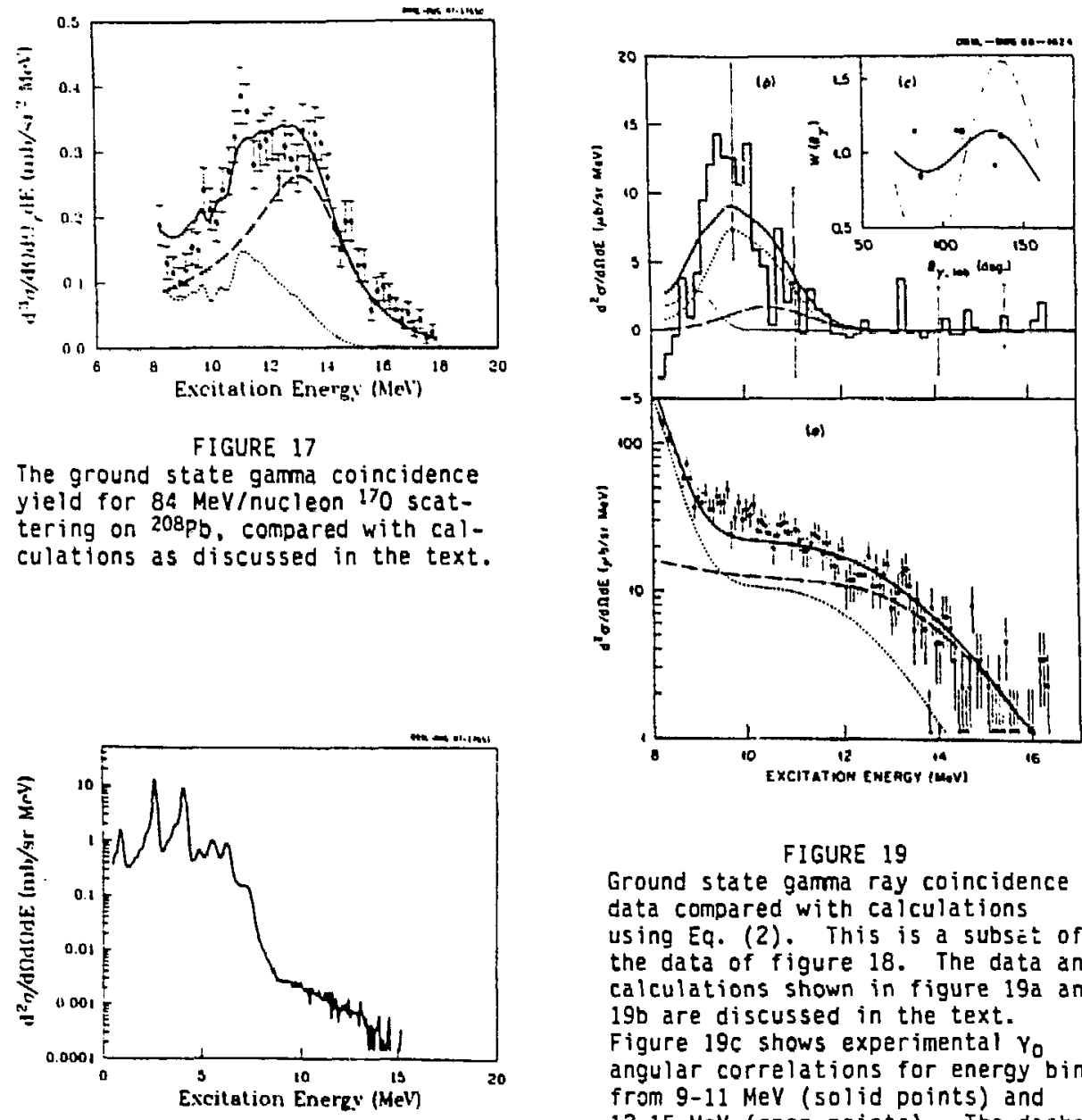

FIGURE 19

The ground state gama coincidence yield for $84 \mathrm{MeV} /$ nucleon 170 scattering on 208pb, compared with calculations as discussed in the text.

FIGURE 18

The angle-integrated ground-state gama ray cross section in coincidence with inelastically scattered 170 ions at $\theta=13^{\circ}$, for $208 \mathrm{~Pb}$ $\left(170,170^{\circ}\right)$ at $22 \mathrm{Mev} /$ nucleon.

Ground state gamma ray coincidence cata compared with calculations using Eq. (2). This is a subset of the data of figure 18 . The data and calculations shown in figure $19 a$ and $19 b$ are discussed in the text. Figure 19c shows experimental $Y_{0}$ angular correlations for energy bins from 9-11 MeV (solid points) and 12-15 MeV (open points). The dashed curve is for pure El decay of the IVGDR. The dash-dot curve is for pure E2 decay of the ISGOR while the solid curve represents the $E 1+E 2$ mixture predicted at 9-11 MeV by the calculations shown in $a$ and $b$. 
decays to the $3^{-}, 2.61$ and $2^{+}, 4.08$ states, respectively. Figure $20 d$ shows the relative strength for decays populating the 4.97-MeV, 3- state. A few of the more striking aspects include the marked absence of strength to the 2.61 and $4.08 \mathrm{MeV}$ states across the resonance region. A strong yield of decays to the 3- state at $4.97 \mathrm{MeV}$ (thought to be a noncollective state dominated by a single $1 p-1$ th configuration) is seen to appear at -9 MeV and remains significant across the GQR region. Table 2 summarizes the data obtained for a 2 MeV wide bin centered on the ISGQR. A particularly interesting feature is the absence of any significant decay directly to the $3^{-}$state at $2.6 \mathrm{MeV}$. Purely statistical arguments suggest that this branch from the region of the ISGQR should be approximately equal to the ground state branch. Clearly it is strongly suppressed. The on!y decays from the ISGQR region strong enough to be clearly

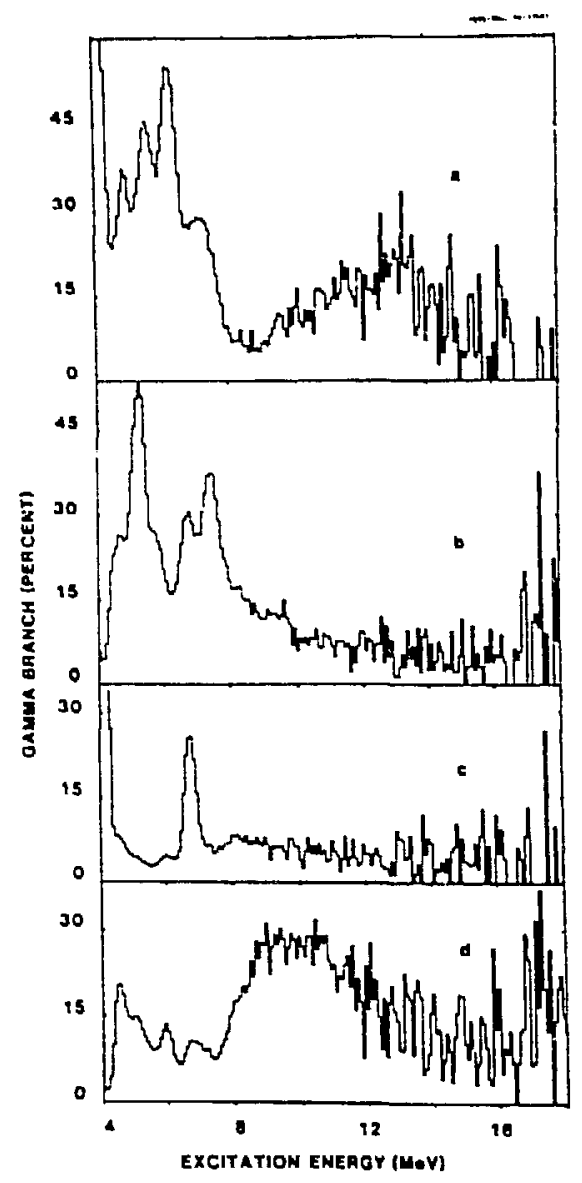
identified are a branch to the 4.9 MeV. $3^{-}$state and a decay to the $5.512 \mathrm{MeV}, 1^{-}$state. The latter is grouped in Table 2 with decays to a number of other states tentatively identified with known !- levels between 5 and $7 \mathrm{MeV}$. The $5.5 \mathrm{MeV}$ state accounts for about $60 \%$ of this yield. Decay to the $5^{-}$state at 3.2 $\mathrm{MeV}$ was seen in the 8.5-9.5 MeV exritation energy bin and weaker evidence for decay to the $5^{-}$state at 3.9 MeV in the 9.5-10.5 MeV bin was found, confirming the presence of $4^{+}$or $6^{+}$strength in these regions. In the decay of the 12.5-15.5 MeV excitation region, the only lines identified correspond to 1- states at $5.51,7.06$, and/or 7.08 MeV. This would be consistent with

FIGURE 20

Relative gamma-decay strengths for transitions to a number of low-lying levels in 207Pb: (a) for ground-state decays; for transitions to the $2.61-\mathrm{MeV}, 3^{-}$state; (c) the 4.08-MeV, $2^{+}$state; (d) the 4.97-MeV, 3- state. 
Table 2

Relative Photon Branching to Low-lying States in $201 \mathrm{~Pb}$

from the Excitation Energy Region of 9.5-11.5 MeV

\begin{tabular}{|c|c|c|c|c|}
\hline \multicolumn{2}{|c|}{ Final state } & \multicolumn{3}{|c|}{ Deras s. ach relative to g.s. } \\
\hline \multirow[t]{2}{*}{ F. $(\operatorname{MeV})$} & \multirow[t]{2}{*}{$j^{\pi}$} & \multirow[t]{2}{*}{ Experiment } & \multicolumn{2}{|c|}{ Calculations } \\
\hline & & & Ref. 12 & Ref. 13 \\
\hline 0.0 & $0^{+}$ & 1.0 & 1.0 & 1.0 \\
\hline 2.61 & $3^{-}$ & $0.04 \pm 0.04$ & 0.027 & 0.035 \\
\hline 4.085 & $2^{+}$ & $\begin{aligned} 0.02 & +0.05 \\
& =0.02\end{aligned}$ & & $9 \times 10^{-3}$ \\
\hline 4.97 & $3^{-}$ & $1.80 \pm 0.50$ & & $2.3^{a}$ \\
\hline $5-7$ & $1-$ & $1.50 \pm 0.50$ & & 0.34 \\
\hline
\end{tabular}

${ }^{a}$ Suin for three states at $4.7,5.5$, and $6.3 \mathrm{MeV}$.

the photon decay mode expected from the giant monopole resonance which cominates the excitation cross section in this region.

The strong suppression relative to statistical estimates of decays from the GQR region to the $2.6 \mathrm{MeV}, 3^{-}$state is very interesting. Two recent calculations ${ }^{12,13}$ (Table 2) predict this suppression. In both calculations the suppression arises from a combination of factors, among which is cancellation between neutron and proton matrix elements because of the isoscalar nature of both the 10.6 MeV quadrupole resonance and the $2.6 \mathrm{MeV}$. 3- state. A significant isovector admixture in the GQR would lead to a strong enhancement of this transition. 13 our data rule out such an admixture. It should be noted that our results on the ground state decay show that compound decay is important for the ISGQR (resulting primarily from unusually small compound neutron widths in 208pb). It would be reasonable to assume that fully damped states are also important in decays to excited states. The suppression of El transitions to the $3^{-}, 2.6 \mathrm{MeV}$ state must therefore survive the damping process, indicating that the compound states into which the GQR mixes retain the isoscalar character of the GQR doorway.

The calculations 12,13 illustrating the sensitivity of the gamma branch from GOR states in $208 \mathrm{pb}$ to the $2.6 \mathrm{MeV}, 3^{-}$state to the isospin character of the 
GQR state was a major motivation for the $84 \mathrm{MeV} /$ nucleon 170 scattering experiments discussed earlier. These calculations suggested that the strong branch to the $3^{-}$state might be an effective way to isolate the IVGQR in 208Pb. The photon decay measurements at GANIL were carried out in an effort to isolate the IVGQR through its unique garma decay. The comparative shortage of information concerning the isovecter GR is largely because of the weakness of isovector excitation in hadron inelastic scattering. The preliminary results of the experiment clearly show how the increasing importance of Coulomb excitation (which is indifferent to isospin) for higher energy heavy-ion scattering changes this situation. There is at least some hape for sensitivity to the IVGQR at GANIL energies, though higher energles would be preferable.

Figure 21 shows the strength distribution for the IVGQR in $208 \mathrm{pb}$ calculated by Bortignon et al. 26 The dashed line in the same figure shows the expected distribution of IVGQR cross section for the $208 \mathrm{pb}(170,1701)$ reaction at 84 Mev/nucleon. resulting from the strong energy dependence of the Coulomb excitation process. The yielc of $\gamma-\gamma$ coincidences involving ciansitions through the $2.6 \mathrm{MeV}, 3^{-}$state should be roughly proportional to the product of these two curves. The histogram in figure 22 shows the $(170,170$ ' $y$ ) data where $E_{Y_{1}}+E_{Y_{2}}=E^{*}$ and $E_{Y_{1}}=2.6 \mathrm{MeV}$. We attribute this spectrum to the IVGQR. The dashed curve shows the folding of the two eurves on figure 21 . The agrement between the data and calculation is striking. Although, as expected, the counts are few, the results are very clean and yleld the values for the centroid, width (sigma), and strength of the IVGQR as shown in Table 3. The results are in excellent agreement with those calculated by Bortignon ${ }^{26}$ and with a recent $(\gamma, n)$ experiment ${ }^{27}$ and not so recent inelastic electron scattering experiments. 28,29

In conclusion we believe that gama decay studies can make a significant contribution to our knowledge of the structure of giant resonances. Heavy ion inelastic scattering followed by gama decay will become an inreasingly important tool at higher bombarding energies. Even for the IVGDR the very large cross sections which can be obtained, and the well understood properties of the dominant Coulomb excitation process should soon supplement or perhaps even improve upon results obtained from photon scattering experiments.

It should be noted that people other than the authors contributed significantly to the various experiments described here. They include R. L. Auble, B. L. Burks, J. Gomez del Campo, M. L. Halbert, D. C. Hensley, J. E. Lisantti, R. L. Robinson, R. O. Sayer, and R. L. Varner from Oak Ridge; W. Mittig and Y. Schutz from GANIL; B. Haas and J. P. Vivien of Strasbourg, and J. Barrette, N. Alamenos, F. Auger, B. Fernandez, and A. Gillibert from Saclay, and A. Nathan from the University of Illinois. 


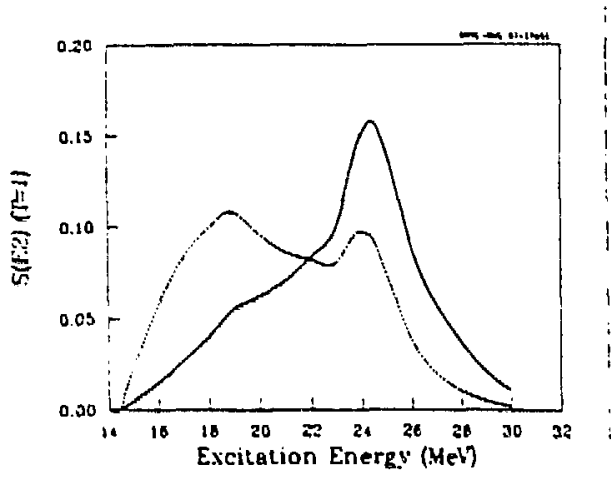

FIGURE 21

The calculzted isovector quadrupole strength function in $208 \mathrm{pb}$ (Ref. 22). The dashed curve is the corresponding distribution of inelastic cross section for $84 \mathrm{MeV} / \mathrm{nucleon} 170$ scattering on 2OEPb.

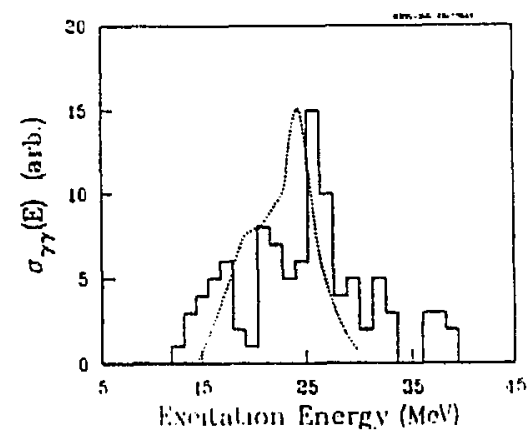

FIGURE 22

The histogram shows the measured relative distribution of $Y Y$ coincidence yield $\left(E_{Y}>10 \mathrm{MeV}\right.$. $E_{y}=2.6 \mathrm{MeV}$ ) as a function of excitation energy, subject to the conditlons discussed in the text. The dashed curve is roughly the product of the two curves in

Fig. 21 .

Table 3

Isovector Giant Quadrupole Resonance $208 \mathrm{~Pb}$

\begin{tabular}{|c|c|c|c|c|}
\hline $\begin{array}{l}\text { Present } \\
\text { Experiment } \\
(170,170 \cdot \mathrm{VY})\end{array}$ & $\begin{array}{l}\text { Bortignon } 26 \\
\text { Calculation }\end{array}$ & $\begin{array}{l}(y, n)^{27} \\
\text { Forward' } \\
\text { Backward Asy. }\end{array}$ & $\left(e, e^{\prime}\right)^{28}$ & $\left(e, E^{\prime}\right)^{29}$ \\
\hline
\end{tabular}

\begin{tabular}{|c|c|c|c|c|c|}
\hline $\begin{array}{l}\text { Centroid } \\
(M \in V)\end{array}$ & $22.6 \pm 0.4$ & 22.4 & $23.5 \pm 1.5$ & -22 & $2 \div .5$ \\
\hline $\begin{array}{l}\text { Hoth } \\
\text { (MEV) }\end{array}$ & $5 \pm 2$ & 3.6 & & & $5 \pm 1$ \\
\hline $\begin{array}{l}\text { EnSR } \\
(\%)\end{array}$ & $-50 \%$ & 61 & & $60 \pm 25$ & $85 \pm 28$ \\
\hline
\end{tabular}




\section{REFERENCES}

1) For a review of these early results see fred E. Bertrand, Nucl. Phys. A354 (1981) $129 c$.

2) J. D. Bowman, H. W. Bạer, R. Bolton, M. D, Cooper, F. H. Cverna, N. S. P. King. M. Leitch, H. S. Matis. A, Erell, J. Alster, A. Doron, M. A. Moinester, E. alackmore, and E. R. Sicilliano, Phys. Rev. Lett. 50 (1983) 1195. A. Erell, J. Alster, J. Lichtenstadt, M. A. Moinester, J. D. Bownair, M. D. Cooper. F. Irom, H. S. Matis, E. Piasetzky, U. Sennhauser, and Q. Ingram. Phys. Rev. Lett. 52 (1984) 2134.

3) For example see N. Frascaria, Y. Blumenfeld, Ph. Chonaz, J. P. Garvon, J. C. Jacmart, J. C. Syynette, and T. Suamyarvi, Nucl. Phys. A474 (1987) 253.

4) F. E. Bertrand, R. O. Sayer, R. L. Auble, M. Geckerman, J. L. Blankenship, B. L. Burks, M. A. G. Fernandes, C. W. Glover, E. E. Gros5, D. J, Horen, J. Gomez del Campo, D. Shapira, and H. P. Morsch, Phys. Rev. C 35 (1987) 111.

5) M. Buenerd, J. Chauvin, G. Dahamel, J. Y. Hostachy, D. Lebrun, P. Martin, P. O. Pellegrin, G. Perrin, and P. de Saintignon, Phys. Lett. 167B (1986) 379.

5) R. Kamermans, J. Van Oriel, H. P. Morsch, J. Wilczynski, and A. van der Woude, Phys, Lett. B82 (1979) 221.

?) T. P. Sjoreen, F. E. Bertrand, R. L. Auble, E. E. Gross, D. J. Horen, D. Shapira, and D. B. Wright, Phys. Rev. C 29 (1984) 1370.

8) F. E. Bertrand, G. R. Satchler, D. J. Horen, J. R. WU, A. D. Bacher, G. T. Emery, W. P. Jones, D. W. Millier, and A. van der Woude, Phys. Rev. C 22 (1980) 1832.

9) F. E. Bertrand, E. E. Gross, D. J. Horen, R. O. Sayer, T. P. Sjoreen, D. K. McDaniels, J. Lisantti, J. R. Tinsley, L. W. Swenson, J. B. MeClelland, T. A. Carey, K. Jones, and S. J. Seestrom-Morris, Phys. Rev. C $34(1986) 45$.

10) J. Barrette, N. Alamanos, F. Auger, B. Fernandez, A. G1llibert, D. J. Horen. J. R. Beene, F. E. Bertrand, R. L. Auble, B. L. Burks, J. Gomez del Campo. M. L. Halbert, R. O. Sayer, W. Mittig, Y. Schutz, B. Haas, and J. P. Vivien, submitled for publication.

11) B. L. Berman and S. C. Fultz, Rev. Mod. Phys. 47 (1975) 713.

12) P. R. Bortignon, R. A. Broglia, and G. F. Bertsch, Phys. Lett. 148B (1984) 20.

13) J. Speth, D. Cha, and V. Klemt, Phys. Rev. C 31 (1985) 2310.

14) J. R. Beene, F. E. Bertrand, M. L. Halbert, R. L. Auble, D. C. Hensiey, D. J. Horen, R. L. Robinson, R. O. Sayer, and T. P. Sjoreen, Nuclear Structure, 1985, edited by R. A. Broglia, G. 8. Hagemann, and B. Herskind, p. 503. 
15) J. R. Beene, F. E. Bertrand, M. L. Halbert, R. L. Auble, D. C. Hensley, D. J. Horen. R. L. Robinson, R. O. Sayer, and T. P. Sjoreen, submitted for publication.

16) J. Raynal, Phys. Rev. C 23 (1961) 2571.

17) G. R. Satchler, Direct Nuclear Reactions (Oxford University Press, Oxford, 1983) and Nucl. Phys. A472 (1987) 215.

18) H. Feshbach, A. Kerman, and S. Koonin, Annals of Physics 125 (1980) 429.

19) M. Hussein and K. McVoy, Phys. Rev. Lett. 43 (1979) 1645 and H. Dias, M. S. Hussein, and S. K. Adhikart, Rev. Lett. 57 (1986) 1998.

20) J. R. Beene, G. F. Bertsch, P. F. Bortignan, and R. A. Broglia, Phys. Lett. 1548 (1985) 19.

21) H. Dias, M. S. Hussein, B. V. Carlson, and A. C. Merchant, Phys, Lett. 1738 (1986) 355.

22) P. A. Moldaver. Phys. Rev, C 11 (1974) 426, P. Axel, K. K. Min, and: D. C. Sutton, Phys. Rev, C 2 T1970) 689 and J. E. Lynn, Theory of Neutron Resonance Cross Sectlons (Oxford University Press, Oxford, 1968).

23) H. P. Morsch, F. Decowski, M. Rogge, P. Turek, L. Zemlo, S. A. Martin, G.P.A. Berg, W. Hürlimann, J. Meissburger, and J.G.M. Römer, Phys. Rev. C 28 (1983) 1947.

24) G. Kilgus, G. Kühner, 5. Müller, A. Richter, and W. Knüpfer, Z. Phys. A326 (1987) 41 .

25) S. J. Seestrom-Morris, C. L. Morris, J. M. Moss, T. A. Carey, D. Drake, J.-C. Dousse, L. C. Bland, and G. S. Adams, Phys. Rev. C 33 (1986) 1847.

¿6) Bortignon, P. R., private comunication.

27) T. Murákami, I. Halpern, D. W. Storm, P. T. Debevec, L. J. Morford, S. A. hiender, and D. H. Dowell, Phys. Rev. C 35 (1987) 479.

28) M. Nagao, Y. Torizuka, Phys. Rev. Lett. 302 (1973) 1068.

29) R. Pitthan, F. R. Buskirk, E. B. Dally, J. N. Dyer, and X. K. Maruyama, Phys. Rev. Lett. 33 (1974) 849; erratum, Phys. Rev. Lett. 34 (1975) 848. 